\title{
Criminologie
}

\section{Trajets urbains et risques de victimisation : les sites de transit et le cas du métro de Montréal}

\section{Marc Ouimet et Pierre Tremblay}

Volume 34, numéro 1, printemps 2001

La notion de risque dans la gestion pénale

URI : https://id.erudit.org/iderudit/004759ar

DOI : https://doi.org/10.7202/004759ar

Aller au sommaire du numéro

\section{Éditeur(s)}

Les Presses de l'Université de Montréal

ISSN

0316-0041 (imprimé)

1492-1367 (numérique)

Découvrir la revue

Citer cet article

Ouimet, M. \& Tremblay, P. (2001). Trajets urbains et risques de victimisation : les sites de transit et le cas du métro de Montréal. Criminologie, 34(1), 157-176. https://doi.org/10.7202/004759ar
Résumé de l'article

Les acteurs urbains sont généralement en mouvement et cessent de l'être seulement lorsque leurs activités exigent d'eux qu'ils demeurent stationnaires pour un intervalle de temps limité. Leurs parcours, composé de sommets (destinations) reliés entre eux par des chemins, forme un circuit, chaque trajectoire ramenant le plus souvent la personne qui se déplace à son point d'origine. Nous analysons la distribution des probabilités individuelles de victimisation personnelle associées aux diverses destinations qui définissent ce parcours (lieux de magasinage, de loisir, de vie domestique et de travail) et ses lieux intercalaires de transit (" la rue », le métro et les autobus). Cette analyse, basée sur les délits rapportés à la police à Montréal en 1995, prend en considération la distribution de la densité, variable selon les sites urbains, des occasions de contacts interpersonnels et propose une évaluation des risques individuels de victimisation auxquels sont exposés les usagers du métro.
Ce document est protégé par la loi sur le droit d'auteur. L'utilisation des services d’Érudit (y compris la reproduction) est assujettie à sa politique d'utilisation que vous pouvez consulter en ligne.

https://apropos.erudit.org/fr/usagers/politique-dutilisation/ 


\title{
Trajets urbains et risques de victimisation : les sites de transit et le cas du métro de Montréal ${ }^{1}$
}

\author{
Pierre Tremblay \\ Professeur \\ École de criminologie \\ Université de Montréal \\ Marc Ouimet \\ Professeur \\ École de criminologie \\ Université de Montréal \\ ouimetm@crim.umontreal.ca
}

RÉSUMÉ - Les acteurs urbains sont généralement en mouvement et cessent de l'être seulement lorsque leurs activités exigent d'eux qu'ils demeurent stationnaires pour un intervalle de temps limité. Leurs parcours, composé de sommets (destinations) reliés entre eux par des chemins, forme un circuit, chaque trajectoire ramenant le plus souvent la personne qui se déplace à son point d'origine. Nous analysons la distribution des probabilités individuelles de victimisation personnelle associées aux diverses destinations qui définissent ce parcours (lieux de magasinage, de loisir, de vie domestique et de travail) et ses lieux intercalaires de transit (" la rue », le métro et les autobus). Cette analyse, basée sur les délits rapportés à la police à Montréal en 1995, prend en considération la distribution de la densité, variable selon les sites urbains, des occasions de contacts interpersonnels et propose une évaluation des risques individuels de victimisation auxquels sont exposés les usagers du métro.

1. Plusieurs personnes ont participé à cette étude et nous tenons à les remercier vivement de leur précieuse collaboration: Jocelyn Grondines, Daniel Brousseau et Isabelle Trottier de la division de recherche et développement de la Société de transport de la Communauté urbaine de Montréal ; Lise Roy et Pierre Ste-Marie de la division d'aménagement urbain de la CUM ; Jean-Denis Ricard du service de surveillance et Serge Bélanger du service d'informatique de la STCUM. Nous souhaitons également remercier Serge Dupont qui a fait en sorte que cette étude puisse être non seulement envisagée mais également réalisée.

Criminologie, vol. 34, $n^{\circ} 1$ (2001) 
ABSTRACT - Origin and destination surveys capture the daily and nightly flows and destinations of work, leisure, shopping and school trips. They provide a basic mobility grid on which to locate crime occurences (reported to the police). In this paper intra-urban trips are assigned various destination points (work, leisure, home, shopping) and paths (streets, metro and bus stations). Place and path-specific rates of personal victimization are then computed on the basis of time spent in such locations and daily prevalence of individuals converging towards them. The paper assumes that the likelihood of being victimized is a multiplicative function of the density of interpersonal contact opportunties. Our main focus is to assess the extent to which actual rates of personal victimization in the metro stations are higher (or lower as, in fact, the findings indicate) than could be expected.

\section{Introduction}

Comme le souligne Felson (1998: 88-90), la mobilité accrue des citadins, combinée notamment avec l'entrée des femmes sur le marché du travail, a eu pour effet de disperser leurs activités quotidiennes loin des lieux résidentiels et de les disperser eux-mêmes dans un plus grand nombre de logements et de véhicules (pour le cas de Montréal, voir Bussière et Dallaire, 1994 ; Coffey et Drolet, 1994 ; Chapleau, 1994). Une conséquence indirecte de cet état de choses a été d'élargir à la fois le rayon d'action des délinquants à la recherche d'occasions criminelles intéressantes et le rayon d'exposition des victimes potentielles : moins d'individus dans les rues susceptibles de prévenir une agression, plus de logements vides durant la journée ou la soirée et plus de véhicules laissés sans surveillance. Une étude récente (Ouimet et al., 1998 : 202-3) indique que plus de la moitié des délinquants arrêtés à Montréal n'habitent pas le quartier où ils ont commis le crime dont on les accuse (cette proportion varie de $32 \%$ à $84 \%$ selon les quartiers et de $34 \%$ à $85 \%$ selon les délits). De manière analogue, une analyse de 1446 délits commis à Philadelphie par une cohorte de délinquants juvéniles (Rand, 1986) montre que les deux tiers d'entre eux ont choisi de commettre leurs délits ailleurs que dans leur quartier de résidence ; même lorsqu'ils réalisaient ces délits à proximité de leur domicile, la moitié des victimes ciblées n'y habitaient pas. D'autres études aboutissent à des résultats analogues (McIver, 1981 ; Gabor et Cottheil, 1984 ; Costanzo et al., 1986 ; Wikstrom et Dolmen, 1990 ; Hesseling, 1992).

Dans cette étude, nous nous intéressons davantage aux déplacements des victimes potentielles qu'aux trajets des délinquants eux-mêmes. Nous supposons que les acteurs urbains sont généralement en mouvement et qu'ils cessent de l'être seulement lorsque leurs activités exigent d'eux qu'ils 
demeurent stationnaires pour une période de temps limitée. Un déplacement est défini comme la décision individuelle de se mouvoir d'un endroit à l'autre pour un motif précis. Deux déplacements qui n'ont pas la même finalité sont considérés comme des déplacements distincts. À chaque déplacement correspond un trajet délimité en amont par son point d'origine (ou de départ) et en aval par son point de destination final. La proportion des citadins qui ne se déplacent pas au moins une fois au cours d'une journée quelconque de la semaine n'a cessé de décliner depuis vingt ans : $21 \%$ en $1938,20 \%$ en $1982,17 \%$ en 1987 et $14 \%$ en 1993 (STCUM, 1995a : 5). Nous tirons profit des sondages origine-destination qui s'intéressent à la séquence précise des trajets urbains journaliers, la nature des moyens de transport utilisés pour chaque déplacement de la séquence, et leurs diverses finalités (magasinage, études, travail, loisirs, retours au domicile). Un déplacement qui utilise plusieurs modes de transport distincts est qualifié de multimodal. Une trajectoire urbaine individuelle est la séquence des déplacements distincts qu'une personne effectue pendant une période de 24 heures. Ce parcours, composé de sommets (destinations) reliés entre eux par des chemins (trajets), forme un circuit, chaque trajectoire ramenant le plus souvent la personne qui se déplace à son point de départ (« retour au domicile»).

La distance ainsi franchie n'est pas seulement physique ou « spatiale ». En se déplaçant, chacun renouvelle la configuration de ses interactions sociales et la position relative qu'il y occupe : il se libère temporairement de l'emprise d'un rapport d'autorité dont il s'éloigne mais d'autres instances régulatrices prendront la relève tout au long du parcours ; il quitte provisoirement un entourage familier, en fréquente d'autres qui lui sont plus étrangers, pour se retrouver sur un « terrain connu » qu'il quittera quelque temps après ; il laisse derrière lui une partie de ses biens, mais s'en procure d'autres en chemin ou à destination, qu'il rapportera ou non au site de départ. Alors qu'il est en mouvement, d'autres le sont également : provenant de milieux sociaux similaires ou très différents, leurs trajets accompagneront le sien ou bien le croiseront de biais ou en sens inverse. Ce sont ces intersections qui définissent la trame sociale de chaque trajectoire individuelle. La densité variable des occasions de contact qui se présente à lui tout au long du parcours détermine en partie la probabilité conditionnelle que ces occasions se développent en rencontres amicales (généralement brèves) ou donnent lieu, au contraire, à des collisions accidentelles ou à des affrontements hostiles. 


\section{Données et stratégie d'analyse}

Les enquêtes origine-destination distinguent habituellement quatre motifs ou destinations de déplacement : le travail et les études, les loisirs, le magasinage et les lieux domestiques (les « retours au domicile »). Par ailleurs les délits rapportés à la police de la Communauté urbaine de Montréal sont classés en fonction du lieu ou du site de leur occurrence. Les rapports d'événements policiers distinguent près d'une centaine « d'endroits » ou « lieux » du crime (cf. Tremblay et Ouimet, 2000 Annexe 2). Nous nous sommes inspirés de la typologie des destinations urbaines pour regrouper les « lieux du crime » mentionnés dans les rapports policiers : les sites domestiques, les sites de travail ou d'étude, les sites de magasinage et les sites de loisirs, d'une part ; et les sites de transit que constituent les rues, les stations de métro et les stations d'autobus, d'autre part.

Nous utilisons la population résidente de la Communauté urbaine de Montréal comme dénominateur de base $(\mathrm{N}=1775779)$ pour calculer les risques de victimisation dans les foyers domestiques et les risques agrégés de victimisation. Pour calculer les risques de victimisation sur la voie publique, nous ajustons à la baisse la population à risque en excluant la proportion de $15 \%$ de citadins immobiles $(\mathrm{N}=1514$ 842). Pour les autres sites urbains (travail-études, magasinage, loisirs) nous utilisons la répartition procentuelle par motif de destination des déplacements fournie par l'Enquête Origine-Destination de 1993 : la proportion des déplacements-destinations sur 24 heures qui convergent vers les lieux de travail ou d'études $(49,1 \%)$, les sites de loisirs $(15,4 \%)$ ou de magasinage $(16,1 \%)$. Cette ventilation par motif de déplacement permet d'apprécier approximativement le nombre de personnes qui fréquentent ces destinations (les données de base pour ces estimations proviennent de la fiche 101 de l'enquête origine-destination réalisée en 1993 par la STCUM, 1995c). La population de référence toutefois, dans ce cas, se limite à ceux qui, à Montréal (CUM), se déplacent effectivement $(\mathrm{N}=1514$ 842). Ainsi le nombre de personnes qui fréquentent les lieux de commerce ou de magasinage est estimé à 243889 (1 514842 $\mathrm{x} 16,1 \%$ ), alors que le nombre de personnes qui fréquentent les destinations de travail ou d'études serait de 743787 (1 514842 x 49,1\%).

Les données de base recueillies par l'enquête sur les déplacements urbains des Montréalais permettent de "déduire » le temps passé en moyenne dans la plupart des sites urbains (Grondines, 1999) : 7,7 heures sur les sites de travail ou d'étude, 3,8 heures dans les destinations de loisir, 2,7 heures dans les sites de magasinage. Les risques de victimisation 
doivent être ajustés en fonction de la durée pendant laquelle les usagers utilisent les sites de transit (sur l'importance de cette composante temporelle dans le calcul des taux de criminalité voir Killias, 1991 : 98-102). Selon l'enquête sur les déplacements urbains des Montréalais réalisée en 1993, chaque usager du métro effectue en moyenne 2 déplacements par jour (722 182 déplacements/367 415 usagers). Le temps moyen passé dans le métro par trajet est de 25 minutes : 4 minutes pour accéder au moyen de transport, 10 minutes de temps d'attente et 11 minutes dans les wagons (Grondines, 1999). Les usagers du métro passent ainsi en moyenne 49 minutes dans le métro par jour (0,83 heures) - une information cruciale pour comparer les risques de victimisation dans le métro et les risques de victimisation dans d'autres sites urbains. Les usagers des autobus consacrent le même temps à leurs trajets. Le temps passé dans les domiciles n'est pas évalué dans cette enquête sur les déplacements urbains : nous le fixons (quelque peu arbitrairement) à 14 heures. De manière analogue, le temps effectivement passé dans la rue n'est pas évalué. Nous lui attribuons provisoirement le même budget-temps qu'aux trajets en transport public mais supposons, en revanche, que tous les citadins (quelles que soient leurs modalités de transport) consacrent 0,83 heure par jour à se promener à pied dans la rue.

Nous nous intéressons dans cette étude au répertoire des délits des attaques et des vols sur la personne qui ciblent les particuliers et qui peuvent se produire indifféremment dans n'importe quel site urbain (site domestique ou site de transit par exemple). Et, parmi les sites de transit, nous évaluons plus spécifiquement les risques de victimisation personnelle auxquels sont exposés les usagers du métro. L'expression «usager du métro » reçoit ici une définition assez stricte : sont ainsi exclus de l'analyse les délits qui ciblent au premier chef les acteurs corporatifs ou institutionnels (vandalisme, mais aussi vols à l'étalage et agressions à l'endroit des agents de la paix), les infractions qui n'impliquent pas de victimes particulières et finalement l'ensemble des victimisations personnelles qui ne sont pas communes à l'ensemble des sites urbains (cambriolage résidentiel par exemple ou vol de véhicule moteur). L'objectif de l'article est également circonscrit : examiner si les risques de victimisation personnelle auxquels sont exposés les usagers du métro sont inférieurs ou supérieurs aux risques comparables (ou équivalents) de victimisation personnelle qu'imposent les autres destinations urbaines.

Les victimisations personnelles qui se produisent dans le métro regroupent les délits commis dans les wagons et ceux qui se produisent 
dans les stations. En combinant ces deux « endroits », il devient possible de recenser le nombre total de délits commis dans le métro. De manière analogue, les délits commis dans les autobus, les abribus et les terminus sont regroupés dans une catégorie distincte (« autobus et terminus »). En pratique, il est malaisé de distinguer un délit commis à proximité du métro et un délit commis dans un terminus à la sortie du métro. Les données les plus détaillées dont nous disposons dans cette étude est la banque informatisée (et dénominalisée) des délits rapportés au Service de police de la Communauté urbaine de Montréal en 1995. En 1995, $86 \%$ des délits commis dans le métro se sont produits ailleurs que dans les wagons proprement dits (i.e. dans les stations ou à proximité) et $52 \%$ des délits commis dans les stations d'autobus ont été commis ailleurs que dans les autobus proprement dits (abribus, terminus). Pour les délits qui sont commis dans le métro, les rapports d'événements consignent souvent, mais pas toujours, la station de métro où ce crime a été commis (qu'il ait été commis dans un wagon ou ailleurs). Dans bien des cas, on ne sait pas exactement où le vol par exemple a été effectivement commis et, dans d'autres cas, l'information est disponible mais n'est pas consignée dans les dossiers. Les délits pour lesquels on connaît la station de métro où ces délits ont été commis forment toujours une fraction du nombre total de délits dont on sait qu'ils ont été commis dans le métro (sans pour autant que l'on puisse identifier la station de leur occurrence). Nous analysons ici le tout et non la fraction.

En 1995, le nombre de victimisations personnelles signalées aux autorités policières qui avaient eu lieu dans le métro de Montréal était de 1690. Le taux de victimisation pour les usagers du métro $(\mathrm{N}=367415)$ est donc de 4,6 par 1000. Ce taux est calculé sur une base annuelle. Pour une journée quelconque celui-ci est de 1,3 sur 100000 (usagers). Il ne représente cependant qu'un indicateur approximatif des risques individuels encourus. L'achalandage journalier du métro a été calculé, en effet, pour une journée moyenne de semaine. En fait, le taux d'achalandage baisse de manière significative le samedi et le dimanche : ainsi la moyenne des déplacements en octobre 1998 était de 321537 les jours de fin de semaine et de 740128 les jours de semaine - une baisse d'achalandage de $57 \%$ (on trouvera dans Tremblay et Ouimet, 2000 une présentation détaillée de ces données). Les victimisations personnelles commises le samedi et le dimanche sont également moins nombreuses (208 en moyenne) qu'en semaine (255 en moyenne) - une baisse relative de $18 \%$. Il convient, par conséquent, d'ajuster à la baisse 
la population quotidienne des usagers du métro de manière à tenir compte de l'achalandage des fins de semaine. La méthode la plus simple est de procéder à une estimation pondérée des usagers du métro (367 415 par jour de semaine et 164024 par jour de fin de semaine). La moyenne pondérée d'achalandage quotidien obtenue est de 309 303. Le taux ajusté de victimisation personnelle dans le métro est ainsi de 5,46 pour 1000 usagers sur une base annuelle et de 1,5 sur 100000 par jour. On peut supposer que la même baisse d'achalandage se produit dans les autres systèmes de transport en commun. Nous utilisons le même facteur de correction (309 303 / 367415 ou 84 \%) pour ajuster à la baisse le volume quotidien d'usagers des autobus (451 263 x $84 \%=379889$ ).

\section{Distribution urbaine des risques de victimisation personnelle}

En 1995, 55292 victimisations personnelles signalées à la police se sont produites à Montréal. Compte tenu de la population urbaine recensée, on obtient donc 152 incidents par jour et un taux de victimisation personnelle de 31 par 1000 résidants. En termes absolus, ce risque est en réalité très faible puisque l'incidence de ces victimisations est calculée sur une base annuelle. Pour une journée quelconque de l'année (152 incidents par jour), ce risque moyen est de 8,5 sur 100000 . On doit également tenir compte du fait que ces risques sont étalés sur 24 heures. Pour comparer les risques de victimisation personnelle d'un site urbain à l'autre (site de destination ou site de transit), il est nécessaire de calculer les risques de victimisation personnelle sur une base horaire. Ainsi les risques agrégés ou totaux de victimisation personnelle par heure d'exposition est de 0,36 par 100 000. (Ce taux est obtenu en divisant le total des victimisations personnelles par $365 \times 24$, en divisant ensuite ce résultat par la population de référence et en l'ajustant par 100 000).

Le tableau 1 compare les risques de victimisation personnelle par site urbain en fonction de l'usage que les citadins en font et en fonction du temps consacré à cet usage. Les résultats de nos analyses (et de nos postulats) sont instructifs à plusieurs égards :

1. La rareté relative des délits qui se produisent dans le métro (1690 seulement dans le métro) ne permet pas de conclure que les risques individuels encourus par les usagers du métro sont triviaux. Alors que les risques généraux de victimisation des résidants de Montréal sont étalés 


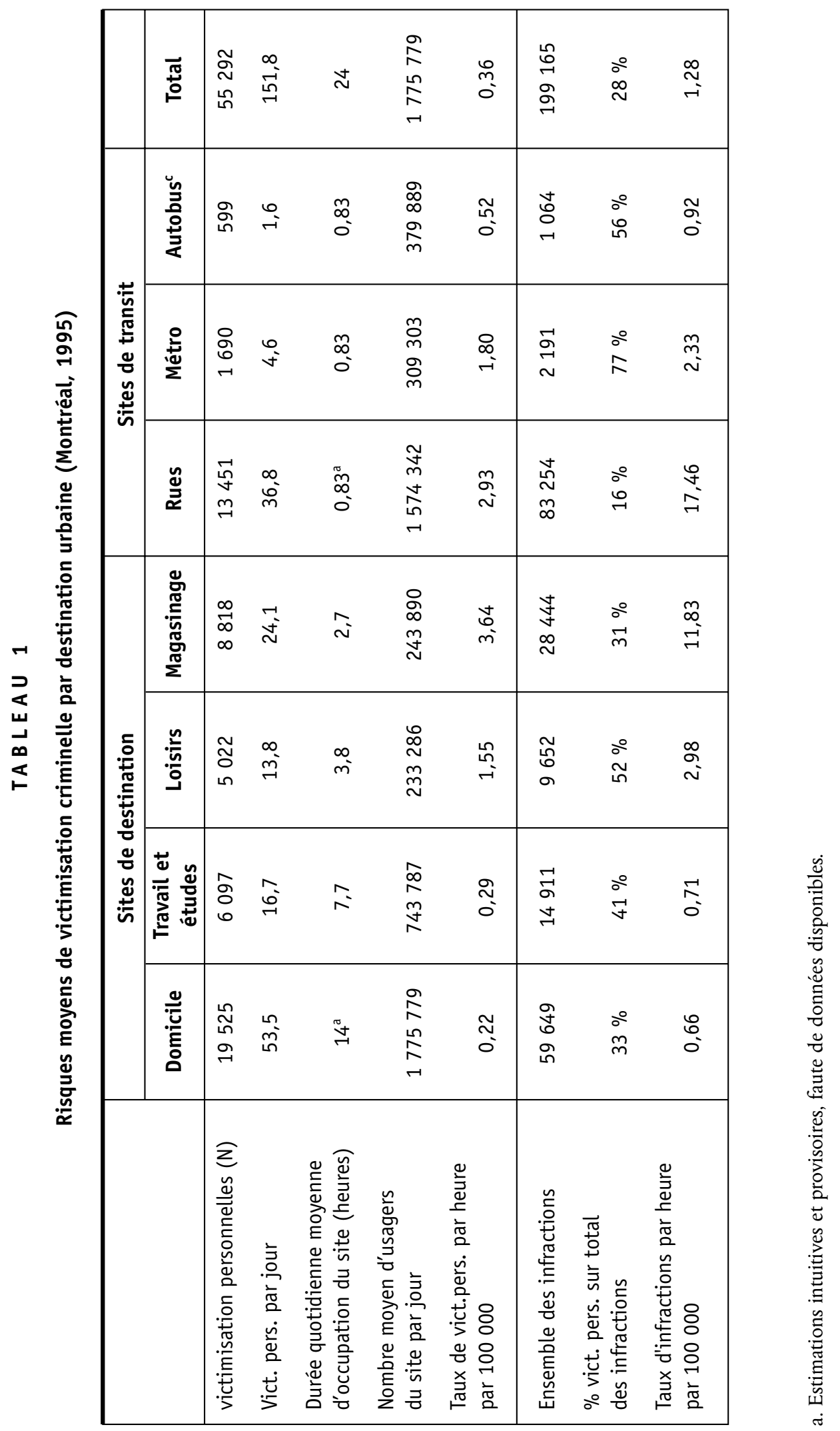


sur 24 heures, le temps passé dans le métro se limite à une période d'environ une heure (49 minutes). Pour estimer les risques de victimisation auxquels sont exposés les usagers du métro, il est préférable de les apprécier en fonction du temps que ces usagers consacrent à ce site urbain : on constate alors que les risques de victimisation personnelle associés à l'usage de ce moyen de transport sont près de six fois plus élevés que le taux agrégé de victimisation personnelle : 1,8 par 100000 par heure (contre 0,36 par 100 000).

2. En réalité, le taux agrégé de victimisation personnelle est largement gouverné par les risques de victimisation personnelle associés aux destinations urbaines auxquelles les citadins consacrent l'essentiel de leur temps (domicile, travail et études). La probabilité individuelle d'être la cible d'un vol sur la personne ou d'une agression est 6 et 8 fois plus élevée dans le métro (1,8 par 100 000) que dans les lieux de travail et les foyers domestiques, mais elle est en revanche inférieure à la probabilité équivalente de victimisation personnelle dans les rues de Montréal (2,9 par 100000$)$, dans les sites de magasinage (3,6 par 100000$)$ et ne differe guère de celle qui prévaut dans les destinations de loisir (1,6 par $100000)$.

3. Les risques de victimisation personnelle varient également selon les réseaux de transport en commun $(0,5$ pour les autobus, 1,8 pour le métro). Ces deux systèmes de transport présentent, cependant, des caractéristiques fort différentes. Les stations de métro présupposent un aménagement de l'espace public beaucoup plus élaboré que celui des stations d'autobus. Pour l'ensemble des délits signalés à la police qui avaient été commis en 1995, 309 ont eu lieu dans les véhicules et 1882 dans les stations ou à proximité ; pour le réseau des transports par autobus, 510 ont eu lieu dans les autobus proprement dits, 416 dans les abribus et 138 dans les édifices de la STCUM. Si l'on se limite aux victimisations personnelles commises dans les véhicules (trains de métro et autobus), les risques de victimisation personnelle semblent comparables et moins prononcés, en fait, dans le réseau du métro que dans celui des autobus $(0,33$ et 0,44 par 100000 respectivement par heure d'exposition)

4. Les vols sur la personne et les agressions commis aux dépens des particuliers ne constituent en effet qu'un sous-ensemble des délits signalés à la police. Ils représentent une proportion élevée des délits commis dans les transports en commun ( $77 \%$ pour les stations de métro, $56 \%$ pour les stations d'autobus) mais une proportion moindre que les 
délits commis dans la rue (16\%), les lieux de magasinage (31\%) ou les domiciles (33\%). Toutefois, la distribution par site urbain des taux de criminalité ne differe pas de manière substantielle de celle des taux de victimisation personnelle : la probabilité d'occurence d'un délit est plus élevée dans les rues (15,48 sur 100000 par heure d'exposition) et les destinations de magasinage (11,83 sur 100 000). Les sites les plus protégés demeurent les foyers domestiques $(0,66$ sur 100000$)$ et les lieux de travail $(0,71$ sur 100000$)$ ainsi que les transports en commun par autobus $(0,92$ sur 100000$)$. Les destinations de loisir (2,98 sur $100000)$ et le métro $(2,33$ sur 100000$)$ présentent une vulnérabilité intermédiaire.

\section{Distribution urbaine des occasions d'interaction}

D’une manière générale, les risque d'agression ou de vol sur la personne augmentent dans les sites où la densité des interactions et des déplacements urbains est élevée (les lieux de magasinage, la voie publique et les corridors de déplacement comme le réseau du métro). Le métro demeure par définition et par vocation un espace de transit et de circulation rapide. Il est donc normal que les occasions d'interaction (de contact ou de rencontre) à la fois bienveillantes, neutres et malveillantes y soient plus nombreuses qu'ailleurs. Le contraire eût été étonnant. La densité des interactions sociales est généralement considérée comme un facteur propice à la délinquance, même s'il n'existe pas de consensus sur la manière d'interpréter cette relation (Tittle, 1989) et que la force ou même la direction de cette relation varie selon la manière dont on opérationnalise et mesure chacun de ses termes (O'Brien, 1983 ; Gillis et Hagan, 1982). Pour les fins de cette étude, nous supposons que la fréquence des victimisations personnelles rapportées à la police est une fonction multiplicative de la densité des interactions interpersonnelles que sont susceptibles de produire les environnements urbains (Mayhew et Levinger, 1976).

Il est pertinent, par conséquent, d'évaluer si les risques de victimisation personnelle observés dans le métro ne sont pas en réalité inférieurs aux risques potentiels ou attendus, compte tenu de la densité très élevée d'occasions d'interaction et de friction qu'offre cet espace de transit. Dans cette analyse, nous estimons la densité des occasions de contact dans quatre environnements : l'île de Montréal dans son ensemble (la 
Communauté urbaine), la ville de Montréal, son centre-ville et le métro lui-même. La densité des occasions de contact est mesurée par le rapport entre le volume des déplacements qui s'effectuent dans un site urbain et la superficie du territoire concerné.

La superficie à vol d'oiseau du métro de Montréal est de $1200 \mathrm{~m}^{2}$ par station. Le réseau inclut 65 stations et sa superficie totale est de $78000 \mathrm{~m}^{2}$. Par contraste, la superficie du territoire du centre-ville de Montréal est de $3,67 \mathrm{~km}^{2}$. Pour fins de comparaison, nous utilisons la superficie du «territoire actif » de la Ville de Montréal $\left(107 \mathrm{~km}^{2}\right.$ sur $\left.171 \mathrm{~km}^{2}\right)$ et de la Communauté urbaine de Montréal $\left(265 \mathrm{~km}^{2}\right.$ sur 487 $\mathrm{km}^{2}$ ), où se localisent les principales destinations des déplacements quotidiens des Montréalais (centre d'achats et commerces de détail, surfaces d'habitation, édifices à bureau, espaces d'utilité publique) ${ }^{2}$.

Les occasions de contact (aimables, hostiles ou indifférents) dans un site urbain dépendent, bien entendu, de plusieurs facteurs : l'achalandage du site, sa superficie, le temps d'occupation de ceux qui s'y rendent, la proportion d'entre eux qui y sont relativement stationnaires une fois rendus, et la vitesse relative de leurs déplacements dans le site. La vitesse de déplacement sur le site dépend du mode de transport dominant et de son achalandage. Le temps d'occupation du site dépend lui-même des motifs pour lesquels les individus s'y rendent (travail, retour au domicile, magasinage, études, loisirs). Il serait souhaitable qu'une modélisation explicite de tous ces facteurs soit entreprise et incorporée dans l'évaluation des risques différentiels de victimisation par site urbain. Une telle modélisation dépasse les limites de cette étude. Nous offrons ici, toutefois, un cadre d'analyse préliminaire pour procéder à une comparaison plus systématique de la densité différentielle des occasions de rencontre dans les divers sites de destination des déplacements urbains et dans les corridors qui sont mis en place pour assurer leur fluidité.

Dans un site de destination (un quartier, une municipalité, l'île de Montréal dans son ensemble), ceux qui se rendent en cet endroit à telle heure s'ajouteront à ceux qui s'y sont rendus durant l'heure précédente et qui y demeurent un certain temps. La densité des occasions de contacts y est donc partiellement cumulative. Dans le métro, par contre, la situation est différente : ceux qui s'y rendent à telle heure remplaceront ceux qui s'y sont rendus durant l'heure précédente. La densité y est rela-

2. Les taux d'occupation du sol en 1996 pour la Ville de Montréal et la Communauté urbaine de Montréal nous ont été aimablement communiqués par Lyse Roy et Pierre SteMarie de la division d'aménagement urbain de la CUM. 
tivement « dissipative ». Les trains de métro se succèdent toutes les 4 minutes durant les heures de pointe et toutes les 11 minutes le reste du temps. Autrement dit, la pulsation avec laquelle le métro se remplit et se désemplit est très rapide, alors que la rotation des flux de population sur les sites de destination y est beaucoup plus lente : le centre-ville se remplit rapidement aux heures de pointe du matin et ne se désengorge que beaucoup plus tard - aux heures de pointe du soir - où se croiseront alors ceux qui retournent à leur domicile et ceux qui viennent s'y divertir pour la soirée ou la nuit. La rotation moyenne des usagers du métro est assurée 7 fois par heure en moyenne pour l'ensemble de la journée (les trains se succédant en moyenne à 8 minutes d'intervalle). Le matin, cette rotation est plus élevée (15 fois par heure).

Pour estimer la densité relative des contacts générés par chaque site urbain, nous utilisons les données relatives au trafic prévalant aux heures de pointe du matin. Nous supposons que ceux qui se rendent à destination y seront stationnaires durant cette période. Pour tenir compte de la nature « dissipative » de la densité humaine dans le métro, nous supposons aussi que le facteur de rotation (ou de remplacement) est 15 fois plus élevé dans le métro que dans les sites urbains de destination (comme le centre-ville ou l'île de Montréal dans son ensemble). Nous supposons enfin que la quantité de déplacements à pied (qui déterminent en dernière instance les occasions de contact direct entre les individus) est proportionnelle à la quantité totale de déplacements urbains qui se produisent dans les sites de destination.

Les résultats de nos analyses (cf. Tableau 2) indiquent que la densité moyenne des occasions de contact et de friction est approximativement 6 fois plus élevée dans l'ensemble des stations de métro que dans le centre-ville ; 28 fois plus élevée que dans la Ville de Montréal dans son ensemble et 39 fois plus élevée que dans la Communauté urbaine de Montréal. (Bien entendu, cette densité des occasions d'interaction et de friction varie considérablement d'une station de métro à l'autre). Cette « sur-densité » des occasions de contact doit être prise en considération lorsqu'on souhaite évaluer jusqu'à quel point les risques de victimisation personnelle observés sont inférieurs ou non aux risques normaux ou prévisibles de victimisation.

D'une part, les risques de victimisation personnelle sont 5 fois plus élevés dans le métro qu'ailleurs à Montréal (CUM) ; en revanche, les occasions de contact (et donc de friction) entre usagers du métro sont 39 fois plus élevées. $S$ 'il est vrai que les victimisations personnelles augmen- 
TABLEAU 2

Densité relative des occasions de contact interpersonnel dans le métro et dans d'autres territoires urbains de Montréal

\begin{tabular}{|l|c|c|c|c|}
\hline & Métro & Centre-Ville & $\begin{array}{c}\text { Ville de } \\
\text { Montréal }\end{array}$ & $\begin{array}{c}\text { Communauté } \\
\text { urbaine de Mtl }\end{array}$ \\
\hline $\begin{array}{l}\text { (1) Superficie totale } \\
\left(\text { en } \mathrm{km}^{2}\right)\end{array}$ & 0,08 & 3,67 & 171,11 & 487,29 \\
$\begin{array}{l}\text { (2) Superficie active } \\
\begin{array}{l}\text { (3) Volume total des } \\
\text { déplacements du matin }{ }^{1}\end{array}\end{array}$ & 0,08 & 3,67 & 107,04 & 265,30 \\
$\quad 349897$ & 185628 & 811184 & 1991364 \\
$\begin{array}{l}\text { (6h00-9h00) } \text { Déplacements par heure } \\
\begin{array}{l}\text { Densité des déplacements } \\
\text { par km² par heure }\end{array}\end{array}$ & 116632 & 61876 & 370395 & 663788 \\
$\begin{array}{l}\text { Densité relative } \\
\text { des contacts (Métro/ } \\
\text { autres territoires) }\end{array}$ & 97194 & 16860 & 3460 & 2502 \\
\hline
\end{tabular}

1. Somme des déplacements-origine et des déplacements-destination (STCUM, 1995 : fiches des secteurs 1,101 et 131 de l'enquête OD).

2. Dans le cas du métro, la densité des déplacement par $\mathrm{km}^{2}$ est ajustée à la baisse par un facteur de 15 rotations par heure.

tent en fonction de l'accessibilité des cibles (et donc de leur densité), on doit par conséquent en conclure que les risques observés sont largement inférieurs aux risques attendus ou prévisibles susceptibles de s'actualiser. En bref, les risques de victimisation personnelle dans le métro seraient environ 8 fois moins élevés qu'ils pourraient l'être, puisque les occasions de contact sont 38 fois plus élevés et que les risques de victimisation personnelle sont seulement 5 fois plus élevés $(38 / 5=7,6)$.

\section{Discussion et conclusion}

Autant la « temporalisation » des taux de victimisation personnelle ajustait à la hausse les risques de victimisation personnelle des usagers du métro, autant la prise en considération explicite de la densité des interactions urbaines permet de conclure que les risques observés sont largement inférieurs aux risques attendus. Une telle conclusion est-elle plausible ? Pourquoi les risques observés de victimisation personnelle dans le métro seraient-ils au départ aussi faibles ? Trois facteurs limitatifs peuvent être 
examinés. Le premier facteur est intrinsèque à la dynamique des interactions humaines que produit naturellement tout réseau de transport en commun. La densité élevée des occasions de contact que l'on trouve dans le métro (ou dans d'autres sites urbains) augmente sans doute les occasions de friction et de victimisation mais elle augmente également les occasions d'entraide ou de " gardiennage » informel entre voyageurs (sur les effets bénéfiques de la densité urbaine, voir Shichor, 1979). La foule offre des occasions criminelles aux délinquants, mais elle protège en même temps les victimes potentielles. Il existe également, sans doute, un seuil critique au-delà duquel la densité ou l'accessibilité de ces cibles excède de toute façon le rayon d'action et de décision des délinquants potentiels et exerce un impact marginal décroissant sur la probabilité qu'une victimisation se produise. Par ailleurs, la grande majorité des délits qui surviennent dans le métro oppose des protagonistes qui ne se connaissent pas. Pour ce qui est des délits dont on peut déterminer (à partir des informations consignées dans les rapports policiers) l'absence ou la présence d'une relation préexistante entre les protagonistes (victimes et délinquants) - et le cas échéant la nature de cette relation (amis, conjoints, connaissances, etc.) seulement $20 \%$ des délits qui sont commis dans le métro opposent des protagonistes qui se connaissaient auparavant. Cette proportion augmente à $35 \%$ pour les délits commis sur la "voie publique », et à $39 \%$ pour ceux qui surviennent dans les lieux de loisir. Elle devient bien entendu prédominante pour les délits commis dans les domiciles (87\%) ou dans les lieux de travail (72\%). Bon nombre de délits résultent ainsi de disputes ou de conflits entre protagonistes qui se connaissent au préalable. L'impersonnalité et la brièveté de la grande majorité des interactions qui se produisent exercent ainsi un effet préventif indirect puisqu'elles restreignent en quelque sorte le répertoire des motivations susceptibles de donner lieu à des frictions susceptibles d'être qualifiées de délits.

Cet état de choses permet d'expliquer, du moins en partie, pourquoi le métro constitue un environnement protecteur pour les femmes (cf. Tremblay et Ouimet, 2000 pour une présentation détaillée de ces données). Les statistiques d'achalandage du métro indiquent que la clientèle féminine représente 55,4\% des usagers, et 41,7\% de Montréal (les hommes représentant $44,6 \%$ des usagers et $48,3 \%$ de la population). Le taux annuel de victimisation personnelle pour les femmes est de 0,86 par 1000 et celui des hommes de 2,72 par 1000, Le ratio de victimisation entre les sexes $(2,72 / 0,86)$ est donc de 3 à 1 : trois fois plus d'hommes que de femmes sont dans le métro la cible d'une attaque ou 
d'un vol sur la personne. Ce qui est vrai du métro est également vrai des autobus. D'autre part, le ratio de victimisation personnelle entre les sexes pour Montréal dans son ensemble peut également être calculé en 1995, puisque nous connaissons le nombre de victimisations personnelles qui touchent les hommes $(\mathrm{N}=11539)$ et les femmes $(\mathrm{N}=11462)$. Le ratio de victimisation personnelle entre les sexes est de 1,11 sur l'ensemble du territoire de Montréal ; la probabilité pour le femmes d'être la cible d'une victimisation personnelle est donc 3 fois moins élevée dans le métro qu'ailleurs à Montréal. Un état de choses qui mérite d'être souligné, car cette vulnérabilité moindre est obtenue même si les vols à la tire (ou de sacs à main) et les vols qualifiés y sont relativement plus fréquents qu'ailleurs.

Un deuxième facteur pourrait expliquer pourquoi les risques de victimisation observés dans le métro sont en deçà des risques attendus. Comme nous l'avons déjà souligné, le répertoire de délits susceptibles d'être commis dans le métro est au départ limité. Les voyageurs laissent derrière eux leurs voitures et leurs maisons - deux cibles majeures des délinquants. Ils voyagent " légèrement » et en cohortes serrées et les gains que les voleurs potentiels peuvent espérer ne sont guère élevés au départ. Le montant médian des vols commis dans le métro a été calculé pour 1275 des 1690 victimisations personnelles commises en 1995. Les résultats sont instructifs : 90 \$ en moyenne par vol pour le métro, $75 \$$ pour les autobus, tandis qu'ils sont de 180 \$ pour l'ensemble des victimisations commises à Montréal (220 \$ pour les victimisations personnelles commises dans la rue, 238 \$ pour celles qui sont commises dans les domiciles, 227 \$ pour les lieux de travail et 200 \$ pour les lieux de loisirs). Les gains que l'environnement du métro offre aux délinquants potentiels sont ainsi relativement modestes au départ. Un bassin limité d'opportunités délinquantes attrayantes contribue à expliquer pourquoi les risques de victimisation sont moins élevés dans le métro qu'ailleurs malgré l'accessibilité des cibles.

Cette interprétation semble d'autant plus probante que la délinquance dans le métro est principalement une délinquance juvénile et que des gains médiocres pour un délinquant adulte le sont beaucoup moins pour un délinquant juvénile. Ils le sont d'autant moins d'ailleurs que la classe d'âge la plus vulnérable aux victimisations personnelles dans le métro a moins de 14 ans (cf. Tremblay et Ouimet, 2000 pour une analyse détaille des risques de victimisation et de délinquance par catégorie d'âge). En effet, les protagonistes impliqués dans les victimisations 
personnelles commises dans le métro sont en majorité des jeunes de 10 à 19 ans : c'est le cas de $52 \%$ des victimes et de $51 \%$ des suspects. Cette proportion est de $16 \%$ seulement pour l'ensemble des victimisations personnelles commises à Montréal. On pourrait supposer que cette dominance de la délinquance juvénile résulte de ce que les 10 à 19 ans ont un poids démographique plus élevé dans le métro que dans d'autres sites urbains. En fait, selon les données d'achalandage de l'enquête origine et déplacement de 1993 (STCUM, 1995b), ils représentent $15 \%$ des usagers du métro et $11 \%$ de la population de Montréal, un écart trop faible pour expliquer la place disproportionnée qu'occupe dans le métro la délinquance juvénile. La criminalité commise dans le métro est dominée par les délinquants de 15 à 19 ans $^{3}$ (taux de délinquance de 6,18 par 1000) et à un moindre degré par les délinquants de 10 à 14 ans (taux de délinquance de 4,40 par 1000). Pour l'ensemble de ces deux groupes d'âge, le taux de délinquance en matière de victimisations personnelles commises dans le métro est de 5,85 par 1000. Quant aux attaques et aux vols sur la personne, elles sont commises principalement au détriment des plus vulnérables ou des plus jeunes, c'est-à-dire les 10 à 14 ans : le taux de victimisation de cette classe d'âge dans le métro est de 9,67 par 1000 — un taux deux fois plus élevé que celui des 15 à 19 ans et neuf fois plus élevé en moyenne que celui des 20 à 54 ans. En fait les 10 à 14 ans constituent la cible principale des attaques commises par les 15 à 19 ans. Les chiffres dont nous disposons suggèrent que le « taxage » qu'exercent les plus « forts » sur les plus « faibles » constitue la configuration dominante de la délinquance juvénile dans les transports en commun. Le fait que les délinquants exploitent un avantage comparatif initial (par exemple leur force physique) est une caractéristique très générale des confrontations délictuelles. Elle n'a rien de spécifique au métro (Farrington, 1993) ou à la délinquance juvénile (Felson, 1996).

Le troisième facteur qui pourrait expliquer pourquoi les usagers du métro sont exposés à des risques de victimisation personnelle très largement inférieurs aux risques théoriques attendus pourrait résulter du fait qu'il s'agit d'un espace urbain fermé qui fait l'objet d'une surveillance

3. Nous connaissons, pour 1995, l'âge des victimes et des suspects impliqués dans 404 incidents. Parmi les 90 victimes âgées de 10 à 14 ans, 25 d'entre elles avaient été attaquées par d'autres écoliers du même âge et 55 par des adolescents de 15 à 19 ans. Parmi les 169 victimes ayant 15 à 19 ans, 126 ont été attaquées par d'autres adolescents du même âge et seulement 32 par des agresseurs plus âgés 
relativement dense. Le nombre de voyageurs dans le métro est de 367415 en moyenne par jour de semaine. Le flux moyen de voyageurs par heure est donc 18371 . On retrouve dans le métro en une heure quelconque de la journée 510 employés (changeurs, opérateurs, hommes d'entretien, etc.) et, parmi ceux-ci, 130 agents de surveillance. Le taux d'encadrement assuré par ces agents de surveillance est ainsi relativement élevé : 7 agents de surveillance par 1000 usagers (130 / 18 371) - un taux d'encadrement beaucoup plus élevé que ne sont capables d'offrir les corps policiers. Bien que la mobilité des agents de surveillance soit limitée (en raison de la dispersion physique des stations de métro et de la lenteur relative de leurs déplacements), le territoire à surveiller est très restreint $\left(78000 \mathrm{~m}^{2}\right.$ plutôt que les $200 \mathrm{~km}^{2}$ qui doivent être patrouillés par les policiers de la Communauté urbaine de Montréal). La densité relative de la surveillance susceptible d'être exercée nous semble considérable.

Ces agents de surveillance bénéficient également des informations et du soutien des autres employés de la STCUM qui y travaillent et de la surveillance informelle qu'ils y exercent. Les statistiques disponibles indiquent la présence physique dans le métro à tout moment d'environ 370 employés en uniforme les jours de semaine. En combinant la surveillance explicite et la surveillance informelle exercée par l'ensemble des employés présents dans le métro, on obtient un taux d'encadrement de surveillance (formelle et informelle) de 27 employés par 1000 usagers. Ces employés portent l'uniforme et l'uniforme est à la fois un symbole d'autorité et la garantie d'un allié officiel en cas de difficultés. Les employés exerceraient ainsi une fonction de dissuasion et de prévention même si leurs tâches précises n'y font pas référence. Le taux de victimisation personnelle oscille entre 0,66 et 0,77 par 1000 usagers durant les jours de semaine mais augmente à 1,20 et 1,37 les samedis et dimanches. Or c'est durant le week-end que les effectifs du personnel du métro baissent d'environ de moitié. De manière analogue, les effectifs des agents de surveillance et du personnel baissent de près de $50 \%$ entre $14 \mathrm{~h} 30$ et $21 \mathrm{~h} 30$ et pourraient contribuer au taux relativement élevé de victimisation personnelle après $18 \mathrm{~h} 00$ (cf. Tremblay et Ouimet, 2001, pour une présentation plus détaillée des données de base). Le nombre de victimisations personnelles par heure diminue en termes absolus en soirée (192 incidents par heure entre 15 heures et 18 heures mais seulement 97 incidents par heure par la suite). Toutefois la baisse d'achalandage diminue environ de moitié en soirée de sorte que le taux de 
victimisation personnelle en soirée demeure élevé $(1,51$ par 1000 usagers comparativement à 1,69 en fin d'après-midi). Nous ne disposons pas de la ventilation horaire de l'achalandage durant la soirée, mais il est vraisemblable que les risques de victimisation augmentent de manière significative après 20 heures. Les délinquants potentiels semblent ainsi réagir à l'intensité de la surveillance exercée par les employés du métro (agents de surveillance inclus). Une analyse plus précise du taux d'encadrement et de surveillance des sites urbains mériterait d'être entreprise. À cette surveillance humaine devrait s'ajouter une analyse de la pénétration, très variable selon les sites urbains également, de la vidéo-surveillance (voir cependant l'impact trivial de cette vidéo-surveillance dans le métro de Montréal au début des années 1990, Tremblay et Grandmaison, 1997).

Une stratégie d'analyse s'évalue en fonction de ses mérites intrinsèques, mais aussi de sa fécondité empirique ou théorique. L'objectif de cette étude n'était pas d'offrir une modélisation formalisée des déplacements urbains et de leur impact sur les risques de victimisation mais d'établir sa pertinence ou son utilité criminologique. Certains paramètres ont été estimés sur une base intuitive (le temps d'occupation des sites domestiques, par exemple, ou le temps moyen passé «dans la rue ») et mériteraient d'être validés. D'un autre côté, la prise en considération explicite des déplacements urbains et de la distribution inégale des occasions d'interactions permet d'offrir une estimation raisonnée de la variabilité topologique des risques de victimisation personnelle selon les destinations urbaines et renouvelle l'analyse de géographie urbaine de la criminalité. Cette approche a permis également de formuler une série d'hypothèses concernant un état de chose que l'analyse conventionnelle des sondages de victimisation et des statistiques policières sont incapables de détecter - en l'occurrence le fait que les risques effectifs de victimisation personnelle auxquels sont exposés les usagers du métro de Montréal sont substantiellement inférieurs aux risques attendus.

\section{Références}

BuSSIÈRE, T. et DALLAIRE, Y. 1994. «Étalement urbain et motorisation : où se situe Montréal par rapport à d'autres agglomérations ? " Cabiers de géographie du Québec 38 (105) : 237-343.

Chapleau, R. 1994. «Les pieds, les pauvres et le métro » Routes et transports 24 (1) : 35-46. 
COFfEY, W.F. et DROLET, R. 1994. « La décentralisation intra-métropolitaine des activités économiques dans la région de Montréal, 1981-1991 »Cabiers de géographie du Québec 38 (105) : 371-394.

Costanzo, C.M., Halperin, W.C. et Gale, N. 1986. «Criminal Mobility and the Directional Component in Journeys to Crime», Pp. 73-96 in Metropolitan Crime Patterns, sous la direction de R.M. Figlio, S.Hakim et G.F. Rengert. Monsey : Willow Tree Press.

FARRINGTON, D.F. 1993. « Understanding and Preventing Bullying » in Crime and Justice: An Annual Review of Research (17), sous la direction de M.Tonry. Chicago : University of Chicago Press.

Felson, M. 1998. Crime and Everyday Life, Thounsand Oaks: Pine Forge Press.

Felson, R.B. 1996. «Big People Hit Little People: Sex Differences in Physical Power and Interpersonal Violence » Criminology 34 (3) : 433-452.

GiLlis, A.R. et HaGAN, J. 1982. « Density, Delinquency and Design: Formal and Informal Control and the Built Environment »Criminology 19 (4) : 514-529.

Gabor, T. et CotTheIL, E. 1984. "Offender Characteristics and Spatial Mobility: An Empirical Study and some Policy Implications » Revue canadienne de criminologie $26: 267-81$.

Grandmaison, R. et Tremblay, P. 1997. «Évaluation des effets de la télésurveillance sur la criminalité commise dans 13 stations du métro de Montréal » Criminologie 30 (1) : 93-110,

Grondines, J. 1999. Profil de la clientèle du métro : durées des activités, Société de transport de la Communauté urbaine de Montréal: Service de la planification et du développement, Division recherche et développement.

Hesseling, R.B.P. 1992. "Using Data on Offender Mobility in Ecological Research " Journal of Quantitative Criminology 8 (1) : 95-112 .

KILlias, M. 1991. Précis de criminologie, Berne : Staempfli.

MAYHEW, B.H. et LEVINGER, R.L. 1976. «Size and Density of Interaction in Human Aggregates » American Journal of Sociology 82 : 86-110.

MCIVER, J.P. 1981. « Criminal Mobility: A Review of Empirical Studies », Pp. 2047 in Crime Spillover, sous la direction de S.Hakim et G.F. Rengert. Beverly Hills : Sage.

O'BRIEN, R.M. 1983. "Metropolitan Structure and Violent Crime: Which Measure of Crime? » American Sociological Review 48 : 434-37.

Ouimet, M., Tremblay, P. et Morselli, C. 1997. Analyse stratégique des facteurs démographiques, économiques et sociaux qui façonnent l environnement du Service de police de la Communauté urbaine de Montréal, Montréal : École de criminologie, Université de Montréal.

SHICHOR, D. 1979. «Population Density and Criminal Victimization: Some Unexpected Findings in Central Cities » Criminology 17 : 184-193.

STCUM 1995a. Faits saillants de PEnquête-Ménages Origine-Destination 1993, Montréal: Société de transport de la Communauté urbaine de Montréal (Direction exécutive - Planification et action commerciale). 
STCUM 1995b. Profil de la clientèle du métro, Montréal : Société de transport de la Communauté urbaine de Montréal (Service de l'action commerciale).

STCUM 1995c. Enquête-Ménages Origine-Destination 1993 : système dinformation agrégésur les enquêtes-ménages de MADITUC, Montréal : Société de transport de la Communauté urbaine de Montréal (Direction exécutive, Planification et action commerciale).

TiTTLE, C.R. 1989. « Urbanness and Unconventional Behavior: A Partial Test of Claude Fisher's Subcultural Theory » Criminology 27 (2) : 273-306.

Tremblay, P. et Ouimet, M. 2000. «Trajets urbains et risques de victimisation » in Cabiers du Centre de recherche sur les transports (CRT 200011). Montréal : École des Hautes Études Commerciales/École Polytechnique, Université de Montréal.

Wikstrom, P.H. et Dolmen, L. 1990. « Crime and Crime Trends in Different Urban Environments » Journal of Quantitative Criminology 6 (1) : 7-28. 